HD-THEP-95-16

\title{
BATALIN-FRADKIN-TYUTIN EMBEDDING OF A SELF-DUAL MODEL AND THE MAXWELL-CHERN-SIMONS THEORY
}

\author{
R. Banerjee* and Heinz J. Rothe \\ Institut für Theoretische Physik \\ Universität Heidelberg \\ Philosophenweg 16, D-69120 Heidelberg
}

\begin{abstract}
We convert the self-dual model of Townsend, Pilch, and Nieuwenhuizen to a first-class system using the generalized canonical formalism of Batalin, Fradkin, and Tyutin and show that gauge-invariant fields in the embedded model can be identified with observables in the Maxwell-Chern-Simons theory as well as with the fundamental fields of the selfdual model. We construct the phase-space partition function of the embedded model and demonstrate how a basic set of gauge-variant fields can play the role of either the vector potentials in the Maxwell-Chern-Simons theory or the fundamental fields of the self-dual model by appropriate choices of gauge.
\end{abstract}

\footnotetext{
* On leave of absence from S.N. Bose Natl. Ctr. for Basic Sc. DB-17, Sec. 1, Salt Lake, Calcutta 700064, India
} 


\section{Introduction}

The self-dual (SD) model in $2+1$ dimensions [1] has been the subject of much discussion [2-4], because of its close connection with the Maxwell-Chern-Simons (MCS) theory [5]. An obvious difference between these two models is that, whereas the MCS-theory is manifestly gauge-invariant, possessing only first class constraints, the dual model is a purely second-class system. However, as was shown in [2], the algebra of the fundamental fields in the SD-model is identical to that of the dual field strengths in the MCS theory. Furthermore the Schwinger defintion of the energy-momentum tensor has an identical structure in both models, provided that in the MCS theory it is expressed in terms of the dual field strengths. This suggests that the SD-model and the MCS theory may just be different gauge-fixed versions of a parent gauge theory. We shall show in this paper that this is indeed the case. Since the MCS theory is manifestly gauge-invariant, the natural starting point for constructing the parent theory is the SD-model. This model can be converted into a first-class system in an extended phase space by following the general ideas of Batalin, Fradkin and Tyutin [6]. Embedding second-class systems into gauge theories has proved to be useful within other contexts [7]. Proceeding in this way, the gauge-invariant fields of the embedded theory are shown in section 2 to be equivalent to the fundamental fields of the SD-model. The strong self-duality constraint in the SD-model, which does not involve the time derivative of the fields, is lifted by the embedding procedure to a weak duality constraint. This constraint turns out to be the generator of the gauge transformations, and hence plays the role of the Gauss law in electrodynamics. The remaining self-duality relations are just the equations of motion. Furthermore the involutive Hamiltonian can be expressed in terms of the gauge-invariant fields, modulo a term proportional to the generator of gauge transformations. This signalizes the existence of an underlying Maxwell-type gauge theory. One is then led in a natural way to the correspondence of the SD model with the gauge-invariant sector of the MCS-theory.

In section 3 we construct the phase-space path integral representation for the embedded model. Here the role of this model as a parent gauge theory becomes most transparent. In the so-called unitary gauge [6] one recovers the partition function of the SD-model, while in another judiciously chosen gauge the partition function of the MCS theory is reproduced. We find that in these gauges a set of basic fields in the parent model alternatively play the role of the fundamental fields in the SD-model and the vector potentials in the MCS theory. We conclude the section by discussing an alternative embedding procedure on the configuration space path integral level by starting from a master Lagrangian which possesses a gauge invariance in all fundamental fields. This further illuminates the interplay between gauge invariance and self-duality previously encountered in the Hamiltonian formulation. 


\section{Embedded Version of the SD-Model}

The Lagrangian of the self-dual model is given by

$$
\mathcal{L}_{S D}=\frac{1}{2} f^{\mu} f_{\mu}-\frac{1}{2 m} \epsilon^{\mu \nu \lambda} f_{\mu} \partial_{\nu} f_{\lambda},
$$

and leads to the field equations

$$
f_{\mu}-\frac{1}{m} \epsilon_{\mu \nu \lambda} \partial^{\nu} f^{\lambda}=0,
$$

which are usually referred to as the self-dual relations [1]. It describes a purely second-class system with the constraints

$$
\begin{gathered}
\Omega_{0} \equiv \pi_{0}{ }^{(f)} \approx 0, \\
\Omega_{i} \equiv \pi_{i}^{(f)}+\frac{1}{2 m} \epsilon_{i j} f^{j} \approx 0, \\
\Omega \equiv f_{0}-\frac{1}{m} \epsilon_{i j} \partial^{i} f^{j} \approx 0,
\end{gathered}
$$

and the canonical Hamiltonian

$$
H_{c}=\int d^{2} x\left[-\frac{1}{2} f^{\mu} f_{\mu}+\frac{1}{m} \epsilon_{i j} f^{0} \partial^{i} f^{j}\right] .
$$

The constraints (2.2b) are a manifestation of the symplectic structure of the Chern-Simons three form appearing in (2.1). Following [8] we shall implement them strongly by using the modified Poisson brackets

$$
\left\{f_{i}(x), f_{j}(y)\right\}=-m \epsilon_{i j} \delta(\vec{x}-\vec{y}), \quad\left(\epsilon_{12}=1\right) .
$$

All other brackets are conventional Poisson brackets. With respect to these we are now left with only two second-class constraints. We next convert the model described by the Hamiltonian (2.3) and the constraints (2.2a) and (2.2c) to a first-class system (with respect to the above mentioned brackets) by following the general ideas of ref. [6]. To this effect we enlarge the original phase space by introducing a canonical pair $\alpha$ and $\pi_{\alpha}$. Then a new set of first-class constraints obtained from the original ones, (2.2a) and (2.2c), is given by

$$
\begin{aligned}
\Omega_{0}^{\prime} & =\Omega_{0}+\alpha, \\
\Omega^{\prime} & =\Omega+\pi_{\alpha} .
\end{aligned}
$$

These constraints are in strong involution. A Hamiltonian which is in involution with $\Omega_{0}^{\prime}$ and $\Omega^{\prime}$ is easily constructed

$$
H^{\prime}=H_{c}+\int d^{2} x\left[\frac{1}{2} \pi_{\alpha}^{2}(x)-\frac{1}{2} \partial^{i} \alpha(x) \partial_{i} \alpha(x)+\alpha(x) \partial_{i} f^{i}(x)\right]
$$


satisfying the involutive algebra

$$
\begin{aligned}
& \left\{\Omega_{0}^{\prime}(x), H^{\prime}\right\}=\Omega^{\prime}(x), \\
& \left\{\Omega^{\prime}(x), H^{\prime}\right\}=0 .
\end{aligned}
$$

Note that $\Omega_{0}^{\prime}$ and $\Omega^{\prime}$ are the generators of the infinitesimal gauge transformations

$$
\begin{aligned}
& \left\{f^{i}(x), G[\theta]\right\}=-\partial^{i} \theta(x), \\
& \{\alpha(x), G[\theta]\}=\theta(x), \\
& \left\{f^{0}(x), G_{0}[\tilde{\theta}]\right\}=\tilde{\theta}(x), \\
& \left\{\pi_{\alpha}(x), G_{0}[\tilde{\theta}]\right\}=-\tilde{\theta}(x),
\end{aligned}
$$

where

$$
\begin{aligned}
& G[\theta]=\int d^{3} x \theta(x) \Omega^{\prime}(x), \\
& G_{0}[\tilde{\theta}]=\int d^{3} x \tilde{\theta}(x) \Omega_{0}^{\prime}(x) .
\end{aligned}
$$

From here we see that the following combinations of fields

$$
\begin{aligned}
& F^{i}=f^{i}+\partial^{i} \alpha, \\
& F^{0}=f^{0}+\pi_{\alpha},
\end{aligned}
$$

are gauge-invariant and satisfy the algebra

$$
\begin{aligned}
& \left\{F^{0}(x), F^{0}(y)\right\}=0, \\
& \left\{F^{0}(x), F^{i}(y)\right\}=\partial^{i} \delta(\vec{x}-\vec{y}), \\
& \left\{F^{i}(x), F^{j}(y)\right\}=-m \epsilon^{i j} \delta(\vec{x}-\vec{y}) .
\end{aligned}
$$

The algebra of the $F^{\mu}$ fields is identical to that of the $f^{\mu}$-fields in the dual model, where all constraints have been implemented strongly [2]. Hence this is true in any gauge. In particular, in the unitary gauge [6], which consists of taking the original second class constraints $(2.2 \mathrm{a})$ and $(2.2 \mathrm{c})$ to be the gauge-fixing conditions, the involutive Hamiltonian, the constraints and the algebra of the (gauge-dependent) fields $f_{\mu}$ become identical to those of the SD-model.

The equation of motion derived from the involutive Hamiltonian (2.6) can be easily shown to have the covariant form

$$
F_{\mu}(x)-\frac{1}{m} \epsilon_{\mu \nu \lambda} \partial^{\nu} F^{\lambda}(x)=0
$$

This is the self-duality relation $(2.1 \mathrm{~b})$ written in the embedded version. The time component of the LHS of this equation is just the generator of time independent gauge transformations. We therefore see that the second class constraint (2.2c) has been lifted in the 
embedded version to a generator of gauge transformations, and therefore plays a similar role as the Gauss operator in quantum electrodynamics. This is further illuminated by expressing the involutive Hamiltonian (2.6) in terms of the gauge-invariant fields $F^{\mu}$ :

$$
H^{\prime}=\frac{1}{2} \int d^{2} x\left[F_{0}^{2}(x)+\vec{F}^{2}(x)\right]-\int d^{2} x f_{0}(x) \Omega^{\prime}(x) .
$$

Although $f_{0}$ appears to play the role analogous to the Lagrange multiplier $A_{0}$ in QED, such a correspondence clearly does not hold for the spatial components $f_{i}$, as is evident from the modified brackets (2.4), which show that $f_{2}$ and $\frac{1}{m} f_{1}$ form a canonical pair. Hence the Hamiltonian (2.12) cannot be that of an ordinary Maxwell theory. However, the gauge symmetry of the embedded model, and the fact that $F_{\mu}$ is divergenceless (as follows from (2.11)), suggest that this gauge symmetry can be exposed in an alternative way by introducing gauge potentials through

$$
F_{\mu}(x)=\epsilon_{\mu \nu \lambda} \partial^{\nu} A^{\lambda}(x)
$$

This is similar in spirit to the work in [9], where the Hopf term could thereby be introduced in the non-linear sigma model. Using (2.13) the Hamiltonian (2.12) takes the form

$$
H^{\prime}=\frac{1}{2} \int d^{2} x\left[\vec{E}^{2}+B^{2}\right]+\frac{1}{m} \int d^{2} x f_{0}(x)(\vec{\nabla} \cdot \vec{E}(x)+m B(x)),
$$

where

$$
\begin{aligned}
E^{i} & =\partial^{i} A^{0}-\partial^{0} A^{i}, \\
B & =-\epsilon_{i j} \partial^{i} A^{j} .
\end{aligned}
$$

The physical states are those which are annihilated by the operator $\vec{\nabla} \cdot \vec{E}(x)+m B$, which is identical to the Gauss operator of the Maxwell-Chern-Simons theory. Furthermore, in the physical space, $H^{\prime}$ is identical with the Hamiltonian of the MCS theory with the correct commutation relations involving $\vec{E}$ and $B$. This follows from the brackets (2.10). We therefore see that the field (2.13) can be identified with the dual field strength tensor in the MCS-theory. Hence by embedding the second-class SD-model, we have arrived in a systematic way at its equivalent formulation in the gauge-invariant sector of the MCS theory with the correspondence

$$
\begin{aligned}
& \left(f_{0}\right)_{S D} \longrightarrow\left(f_{0}+\pi_{\alpha}\right)_{e m b} \longrightarrow\left(F_{0}\right)_{M C S} \\
& \left(f_{i}\right)_{S D} \longrightarrow\left(f_{i}+\partial_{i} \alpha\right)_{e m b} \longrightarrow\left(F_{i}\right)_{M C S}
\end{aligned}
$$

where the subscript "emb" stands for "embedded model".

We conclude this section with some observations. The action of the SD-model possesses no gauge symmetry, while the equations of motion are the self-dual relations. In the 
embedded version the theory has a gauge invariance but the fields $f_{\mu}$ no longer satisfy an equation of motion, having the self-dual form. The self-duality relation is recovered in the gauge-invariant sector which involves a combination of the basic fields. Correspondingly the (gauge invariant) dual field strengths (2.13) in the MCS theory satisfy the self-dual relations (2.11) (which are just the usual MCS equations of motion written in terms of the dual fields), whereas this is not the case for basic gauge-variant fields $A_{\mu}$. This illustrates the interplay between gauge invariance and self-duality in these models.

\section{Path Integral Approach}

In the previous section we have studied the connection between the SD- model and the MCS- theory within the Hamiltonian framework by converting the second-class SD- model into a first-class system in an extended phase space. The purpose ot this section is twofold. We first construct the phase space partition function of the embedded model considered in the previous section and show how by appropriate choices of gauges one recovers either the SD model or the MCS- theory. Thereafter we shall consider a purely configuration space path integral approach starting from a master Lagrangian, differing from those previously studied in the literature $[2-4,10]$. This will complement the Hamiltonian analysis carried out in the previous section.

Consider the involutive Hamiltonian (2.6) with the canonical pairs given by $\left(f_{0}, \pi_{0}\right),\left(\alpha, \pi_{\alpha}\right)$ and $\left(f_{2}, \frac{1}{m} f_{1}\right)$. The phase space partition function is therefore given by

$$
Z=\int D f_{0} D \pi_{0} D f_{i} D \alpha D \pi_{\alpha} \delta\left(\Omega_{o}^{\prime}\right) \delta\left(\Omega^{\prime}\right) \delta\left(G_{i}\right) e^{i \int d^{3} x\left[\pi_{0} \dot{f}_{0}+\frac{1}{m} f_{1} \dot{f}_{2}+\pi_{\alpha} \dot{\alpha}-H^{\prime}\right]},
$$

where $\Omega_{0}^{\prime}$ and $\Omega^{\prime}$ are the first-class constraints (2.5), $H^{\prime}$ is the involutive Hamiltonian (2.6), and $G_{i}(i=1,2)$ are two gauge-fixing functions which we shall take to be linear in the fields. Hence the Faddeev-Popov determinant is trivial and has not been included. Taking the original second-class constraints (2.2a) and (2.2c) as the unitary [6] gauge-fixing conditions we obtain

$$
Z=\int D f_{\mu} \delta\left(f_{0}-\frac{1}{m} \epsilon_{i j} \partial^{i} f^{j}\right) e^{i \int d^{3} x \mathcal{L}_{S D}},
$$

where $\mathcal{L}_{S D}$ is the Lagrangian (2.1a) of the SD-model. This is the configuration-space partition function which one obtains from a conventional phase-space analysis.

Alternatively, consider the gauge

$$
G_{i}=f_{i}-\frac{1}{m} \epsilon_{i j} \partial^{j} f_{0}=0 .
$$

Performing the (trivial) momentum integrals one finds

$$
Z=\int D f_{\mu} D \alpha \delta\left(f_{i}-\frac{1}{m} \epsilon_{i j} \partial^{j} f_{0}\right) e^{i \int d^{3} x \mathcal{L}},
$$


where

$$
\mathcal{L}=-\frac{1}{4 m^{2}} f^{i j} f_{i j}+\frac{1}{2 m} \epsilon^{i j} f_{i} \partial_{0} f_{j}+\frac{1}{2} f^{i} f_{i}+\frac{1}{2} \alpha \vec{\nabla}^{2} \alpha-\frac{1}{m} \alpha \epsilon_{i j} \partial^{i} f^{0 j}
$$

with

$$
f_{\mu \nu}=\partial_{\mu} f_{\nu}-\partial_{\nu} f_{\mu}
$$

The $\alpha$ - integral is readily performed. Making use of $\partial_{i} f_{i}=0$ and $\partial_{i} f_{0}=-m \epsilon_{i j} f^{j}$, following from the gauge condition (3.3), one finds after some algebra

$$
Z=\int D A_{\mu} \delta\left(A_{i}-\frac{1}{m} \epsilon_{i j} \partial^{j} A_{0}(x)\right) e^{i \int d^{3} x \mathcal{L}_{M C S}}
$$

where

$$
A_{\mu}=\frac{1}{m} f_{\mu}
$$

and

$$
\mathcal{L}_{M C S}=-\frac{1}{4} F_{\mu \nu} F^{\mu \nu}+\frac{m}{2} \epsilon_{\mu \nu \lambda} A^{\mu} \partial^{\nu} A^{\lambda}
$$

is the Lagrangian of the familiar Maxwell-Chern-Simons theory [5]. We now recognize that the gauge-fixing conditions (3.3) are equivalent to choosing the Coulomb gauge and $\nabla^{2} A^{0}=m \epsilon^{i j} \partial_{i} A_{j}$, which is just Gauss' law in this gauge. Hence $f_{\mu}$ now plays the role of the vector potential in the MCS theory, while in the unitary gauge $f_{\mu}$ was the fundamental field of the SD model. In a general gauge, on the other hand, the gaugeinvariant combination $f_{\mu}+\partial_{\mu} \alpha$ (with $\dot{\alpha}$ defined through the Hamiltonian equations of motion) plays the role of the self-dual field in the SD model, or the dual field strength $F_{\mu}=\epsilon_{\mu \nu \lambda} \partial^{\nu} A^{\lambda}$ in the MCS theory.

This completes the phase space path integral analysis starting from the involutive Hamiltonian of the embedded model. We conclude this section with a pure configuration space path integral analysis, which will further elucidate the interplay between gauge invariance and self-duality referred to earlier.

Consider the following master Lagrangian

$$
\mathcal{L}=-\frac{1}{4} F_{\mu \nu} F^{\mu \nu}+\epsilon_{\mu \nu \lambda} f^{\mu} \partial^{\nu} A^{\lambda}-\frac{1}{2 m} \epsilon_{\mu \nu \lambda} f^{\mu} \partial^{\nu} f^{\lambda}
$$

where $F_{\mu \nu}=\partial_{\mu} A_{\nu}-\partial_{\nu} A_{\mu}$. In contrast to the master Lagrangians considered in refs. $[2,10]$ the action corresponding to $(3.6)$ is independently gauge-invariant under the transformations $A_{\mu} \rightarrow A_{\mu}+\partial_{\mu} \Lambda$, and $f_{\mu} \rightarrow f_{\mu}+\partial_{\mu} \lambda$. The generating functional for the gauge-invariant fields $\epsilon_{\mu \nu \lambda} \partial^{\nu} A^{\lambda}$ and $\epsilon_{\mu \nu \lambda} \partial^{\nu} f^{\lambda}$ is given by

$$
Z[j, J]=\int D A_{\mu} D f_{\mu} \delta\left(\partial_{\mu} A^{\mu}\right) \delta\left(\partial_{\mu} f^{\mu}\right) e^{i \int d^{3} x\left[\mathcal{L}+\left(\epsilon_{\mu \nu \lambda} \partial^{\nu} A^{\lambda}\right) J^{\mu}+\frac{1}{m}\left(\epsilon_{\mu \nu \lambda} \partial^{\nu} f^{\lambda}\right) j^{\mu}\right]}
$$


where a covariant gauge has been chosen for both the $A_{\mu}$ and $f_{\mu}$ fields. Performing first the integration over $f_{\mu}$, implementing the gauge condition $\partial_{\mu} f^{\mu}=0$ by t'Hooft's method, one obtains

$$
Z[j, J]=\int D A_{\mu} \delta\left(\partial_{\mu} A^{\mu}\right) e^{i \int d^{3} x\left[\mathcal{L}_{M C S}+\frac{1}{2 m} \epsilon_{\mu \nu \lambda} j^{\mu} \partial^{\nu} j^{\lambda}+\epsilon_{\mu \nu \lambda} \partial^{\nu} A^{\lambda}\left(j^{\mu}+J^{\mu}\right)\right]},
$$

where $\mathcal{L}_{M C S}$ is the Lagrangian of the Maxwell-Chern-Simons theory (3.5c). For vanishing sources this is the partition function of the MCS theory in a covariant gauge.

Alternatively, performing the $A_{\mu}$ integration in an analogous way, one finds that

$$
Z[j, J]=\int D f_{\mu} \delta\left(\partial_{\mu} f^{\mu}\right) e^{i \int d^{3} x\left[\mathcal{L}_{S D}+\frac{1}{2} J_{\mu}\left(g^{\mu \nu}-\frac{\partial^{\mu} \partial^{\nu}}{\square}\right) J_{\nu}+f_{\mu} J^{\mu}+\tilde{f}_{\mu} j^{\mu}\right]}
$$

where $\mathcal{L}_{S D}$ is given by (2.1a) and $\tilde{f}_{\mu}$ is the dual of $f_{\mu}$ :

$$
\tilde{f}_{\mu}=\frac{1}{m} \epsilon_{\mu \nu \lambda} \partial^{\nu} f^{\lambda} .
$$

Notice that for vanishing sources (3.9) does not reduce to the partition function of the SDmodel, because of the non-trivial measure. In fact, as we now show, the $f_{\mu}$-field appearing in (3.9) does not satisfy the self-duality relation (2.1b) on the level of Green functions. Differentiating in turn the equivalent expressions (3.8) and (3.9) with respect to $J^{\mu}$ and $J^{\nu}, J^{\mu}$ and $j^{\nu}$, and $j^{\mu}, j^{\nu}$, one finds

$$
\begin{aligned}
<F_{\mu}(x) F_{\nu}(y)>_{M C S} & =<f_{\mu}(x) f_{\nu}(y)>-i\left(g_{\mu \nu}-\frac{\partial_{\mu} \partial_{\nu}}{\square}\right) \delta(x-y) \\
& =<f_{\mu}(x) \tilde{f}_{\nu}(y)>=<\tilde{f}_{\mu}(x) \tilde{f}_{\nu}(y)>-\frac{i}{m} \epsilon_{\mu \nu \lambda} \partial^{\lambda} \delta(x-y)
\end{aligned}
$$

From (3.10) we see that, because of the presence of a non-local propagating term, $f_{\mu}(x)$ cannot be identified with $\tilde{f}_{\mu}(x)$, and therefore also not with the self-dual field appearing in (2.1). Indeed, as we now demonstrate, it plays a role analogous to that of the $f_{\mu}$-field in the embedded version of SD-model. To this effect we express the $\delta$-function in (3.9a) as a Fourier transform with Fourier variable $\beta(x)$. One then finds that (3.9a) can be written in the form

$$
\begin{aligned}
& Z[j, J]= \\
& \int D f_{\mu} D \beta e^{i \int\left\{\mathcal{L}_{S D}\left[f_{\mu}+\partial_{\mu} \beta\right]-\frac{1}{2} \partial_{\mu} \beta \partial^{\mu} \beta+\frac{1}{2} J_{\mu}\left(g^{\mu \nu}-\frac{\partial^{\mu} \partial^{\nu}}{\square}\right) J_{\nu}+\left(f_{\mu}+\partial_{\mu} \beta\right) J^{\mu}+\frac{1}{m}\left[\epsilon_{\mu \nu \lambda} \partial^{\nu}\left(f^{\lambda}+\partial^{\lambda} \beta\right)\right] j^{\mu}+\beta \partial_{\mu} J^{\mu}\right\}}
\end{aligned}
$$

where

$$
\mathcal{L}_{S D}\left[f_{\mu}+\partial_{\mu} \beta\right]=\frac{1}{2}\left(f_{\mu}+\partial_{\mu} \beta\right)\left(f^{\mu}+\partial^{\mu} \beta\right)-\frac{1}{2 m} \epsilon_{\mu \nu \lambda}\left(f^{\mu}+\partial^{\mu} \beta\right) \partial^{\nu}\left(f^{\lambda}+\partial^{\lambda} \beta\right) .
$$


Introducing the new fields

$$
h_{\mu}(x)=f_{\mu}(x)+\partial_{\mu} \beta(x)
$$

and carrying out the integration over $\beta$ yields

$$
Z[j, J]=\int D h_{\mu} e^{i \int d^{3} x\left\{\mathcal{L}_{S D}[h]+\frac{1}{2} J_{\mu} J^{\mu}+h_{\mu} J^{\mu}+\frac{1}{m}\left(\epsilon_{\mu \nu \lambda} \partial^{\nu} h^{\lambda}\right) j^{\mu}\right\}} .
$$

By performing the appropriate differentiations with respect to the sources $j^{\mu}$ and $J^{\mu}$ of the equivalent representations (3.8) and (3.13) we obtain relations analogous to (3.10):

$$
\begin{aligned}
<F_{\mu}(x) F_{\nu}(y)>_{M C S} & =<h_{\mu}(x) h_{\nu}(y)>_{S D}-i g_{\mu \nu} \delta(x-y) \\
& =<h_{\mu}(x) \tilde{h}_{\nu}(y)>_{S D} \\
& =<\tilde{h}_{\mu}(x) \tilde{h}_{\nu}(y)>_{S D}-\frac{i}{m} \epsilon_{\mu \nu \lambda} \partial^{\lambda} \delta(x-y) .
\end{aligned}
$$

From here we can conclude that, apart from non-propagating contact terms, the following identifications hold on the level of Green functions

$$
h_{\mu}(x) \leftrightarrow \tilde{h}_{\mu}(x) \leftrightarrow F_{\mu}(x)
$$

These are just the known correspondences $[2,4]$ among the fundamental fields in the SDmodel, and the dual field strengths in the MCS theory. The gauge-invariant field $h_{\mu}(x)$ is the Lagrangian version of the gauge-invariant combinations $f_{0}+\pi_{\alpha}, f_{i}+\partial_{i} \alpha$ in the embedded model discussed in section 2 .

\section{Conclusion}

In this paper we have applied the general ideas of Batalin-Fradkin-Tyutin [6] to obtain a deeper insight into the connection between the fields in the self-dual model and those in the Maxwell-Chern-Simons theory. By embedding the self-dual model in an extended phase space, the fields in the gauge-invariant sector could be identified on one hand with the dual field strengths in the MCS theory and on the other hand with the fundamental fields of the SD-model. As a consequence, the equations of motion for these fields (including the constraint) are just the self-dual relations.

By studying the phase-space partition function corresponding to the involutive Hamiltonian of the embedded model in two particular gauges, we recovered the configuration space partition functions of the SD-model as well as that of the MCS-theory. The $f_{\mu}$ field in the embedded model could be identified with either the fundamental field in the SDmodel or the gauge potentials in the MCS theory. In this sense the involutive Hamiltonian 
played the role of a parent Hamiltonian, and the $f_{\mu}$ field, in the embedded model, that of an interpolating field.

Finally we have discussed an embedding procedure on the configuration space path integral level, differing from the Stückelberg [11] embedding, by starting from a master Lagrangian which, in contrast to the ones considered in the literature $[2,10]$, has a gauge invariance in all fundamental fields. Apart from revealing the common origin of the SDmodel and MCS theory, it provided an instructive way of exhibiting the interplay between gauge invariance and self-duality.

\section{Acknowledgments}

One of the authors (R.B) would like to thank the Alexander- von- Humboldt Foundation for providing the financial support which made this collaboration possible.

\section{References}

[1] P.K. Townsend, K. Pilch and P. van Nieuwenhuizen, Phys. Lett. B136, (1984), 38; ibid, B137, (1984), 443 (Addendum).

[2] S. Deser and R. Jackiw, Phys. Lett. B139, (1984), 371.

[3] E. Fradkin and F.A. Schaposnik, Phys. Lett. B338, (1994), 253.

[4] R. Banerjee, H.J. Rothe and K.D. Rothe, "On the Equivalence of the Maxwell- ChernSimons Theory and a Self- Dual Model", Heidelberg Preprint (HD- THEP- 95- 13).

[5] S. Deser, R. Jackiw and S. Templeton, Phys. Rev. Lett. 48, (1982), 975; Ann. Phys. (NY) 140, (1982), 372.

[6] I.A. Batalin and E.S. Fradkin, Nucl. Phys. B279, (1987), 514; I.A. Batalin and I.V. Tyutin, Int. J Mod. Phys. A6, (1991), 3255.

[7] T. Fujiwara, Y. Igarashi and J. Kubo, Nucl. Phys. B341, (1990), 695; Y.W. Kim, S.K. Kim, W.T. Kim, Y.J. Park, K.Y. Kim and Y. Kim, Phys. Rev. D46, (1992), 4574; R. Banerjee, Phys. Rev. D48, (1993), R5467; R. Banerjee, H.J. Rothe and K.D. Rothe, Phys. Rev. D49, (1994), 5438; Nucl. Phys. B426, (1994), 129; N. Banerjee, R. Banerjee and S. Ghosh, Phys. Rev. D49, (1994), 1996; Nucl. Phys. B417, (1994), 257.

[8] L.D. Faddeev and R. Jackiw, Phys. Rev. Lett. 60, (1988), 1692.

[9] F. Wilczek and A. Zee, Phys. Rev. Lett. 51, (1983), 2250.

[10] A. Karlhede, U. Lindstrom, M. Rocek and P. van Nieuwenhuizen, Phys. Lett. B186, (1987), 96.

[11] E.G. Stuckelberg, Helv. Phys. Acta 30, (1957), 209. 